\title{
Performance of the forward calorimeters for heavy-ion experiments at FAIR, NICA, and CERN SPS
}

\section{Mikhaylov*i}

Nuclear Physics Institute, Czech Academy of Sciences, Řež, Czech Republic

Czech Technical University in Prague, Faculty of Nuclear Sciences and Physical Engineering,

Prague, Czech Republic

National Research Tomsk Polytechnic University, Department of Electronics and Automation of

Nuclear Plants, Tomsk, Russia (on leave from)

\section{F. Guber, A. Ivashkin}

Institute for Nuclear Research, Russian Academy of Science, Moscow, Russian Federation

\section{A. Kugler, S. Kushpil, V. Kushpil, O. Svoboda, P. Tlustý}

Nuclear Physics Institute, Czech Academy of Sciences, Řež, Czech Republic

\section{Ladygin}

Joint Institute for Nuclear Research, Laboratory of High Energy Physics, Dubna, Russia

\section{S. Seddiki}

GSI Helmholtzzentrum für Schwerionenforschung GmbH, Darmstadt, Germany

\section{Selyuzhenkov}

GSI Helmholtzzentrum für Schwerionenforschung GmbH, Darmstadt, Germany

National Research Nuclear University MEPhI, Moscow, Russia

The Projectile Spectator Detectors (PSDs) for the NA61/SHINE at CERN SPS and the CBM at FAIR, and Zero Degree Calorimeter (ZDC) for the MPD at NICA are discussed. The PSDs and ZDC are compensating lead-scintillator calorimeters designed to measure the energy distribution of the forward going projectile nucleons and nuclei fragments (spectators) produced close to the beam rapidity. Design of the PSD modules, their readout electronics, and calirmeter performance for the collision centrality and reaction plane determination are presented. The PSD module tests with different beams at CERN SPS and results of radiation hardness tests of the avalanche photodiodes used for light readout from the PSD module are also reported.

The European Physical Society Conference on High Energy Physics

22-29 July 2015

Vienna, Austria

\footnotetext{
${ }^{*}$ Speaker.

†email: mikhaylov@ujf.cas.cz
} 


\section{Overview of forward calorimeters for FAIR, NICA, and CERN SPS experiments}

A concept of the calorimeter which registers hadrons via their strong interaction with the detector material was for a first time used in late 1950s in a cosmic ray measurements [1]. The calorimeter response can be described in terms of the ratio between the electromagnetic $(e)$ and hadronic $(h)$ components of the shower created inside the detector material. A compensating calorimeter is the one which approaches the compensating condition of $e / h \approx 1$. In these proceedings the compensating forward calorimeters designed for the NA61/SHINE at CERN SPS, the CBM at FAIR, and the MPD at NICA are discussed. These forward calorimeters are designed to detect projectile spectators, i.e. the non-interacting nucleons and fragments emitted at very low polar angles in forward direction in nucleus-nucleus collisions. They are used to determine the centrality of a heavy-ion collision and estimate the orientation of the reaction plane for anisotropic flow measurements.

Calorimeters are segmented transversely, which allows to measure the direction and energy of the registered particles, and longitudinally, which provides information about the shower development inside the detector.
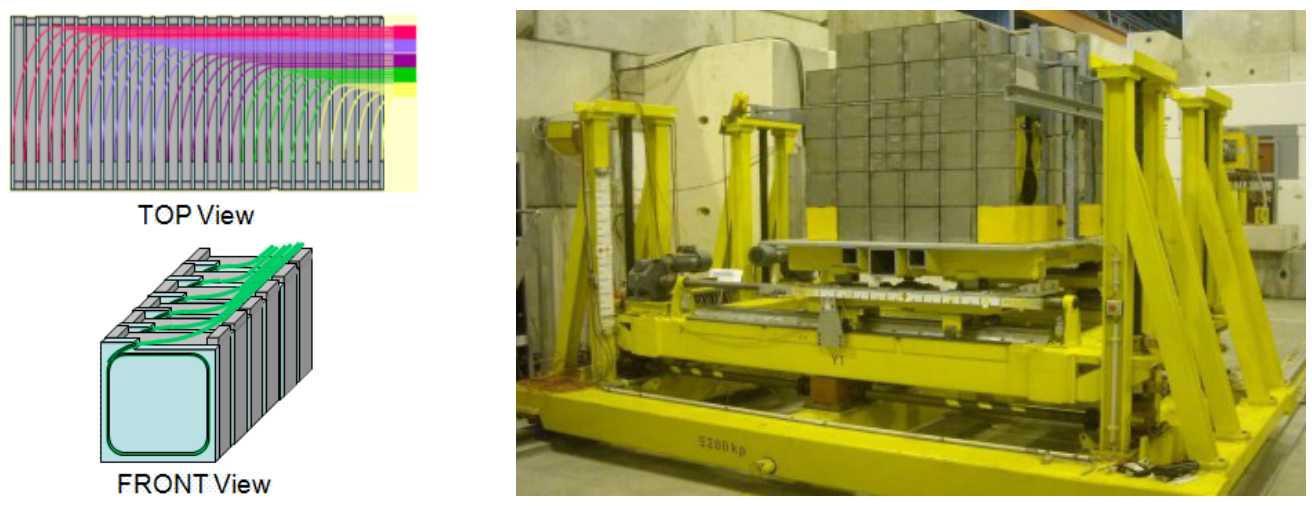

Figure 1: (left) Module design of the forward calorimeter. (right) An image of the NA61/SHINE PSD.

The module, which is the main element of the NA61/SHINE, CBM, and MPD forward calorimeters, is shown in Fig. 1(left). The module design includes lead-scintillator sandwich with a group of consecutive scintillator layers read out by a single photon detector via the wave-length shifting (WLS) fibers. The sections with the individual light readout provide the longitudinal segmentation of the module. The light from the WLS-fibers is readout with avalanche photodiodes (APDs). The main differences between the NA61/SHINE, CBM, and MPD calorimeters are in the transverse cross-section of the modules and their number (detector segmentation), layout in the plane transverse to the beam, and readout electronics.

The Projectile Spectator Detector (PSD) of the NA61/SHINE [2] consists of 44 modules, which cover a transverse area of $120 \times 120 \mathrm{~cm}^{2}$ (Fig. 1(right)). The central part of the NA61/SHINE PSD consists of 16 small modules with transverse dimension of $10 \times 10 \mathrm{~cm}^{2}$, the outer part consists of 28 large $20 \times 20 \mathrm{~cm}^{2}$ modules (transverse layout of modules is shown in Fig. 2(left)). Each module consists of 60 pairs of alternating lead plates and scintillator tiles with $16 \mathrm{~mm}$ and $4 \mathrm{~mm}$ thickness, respectively. The stack of plates is tied together with $0.5 \mathrm{~mm}$ thick steel tape and placed in a box made of $0.5 \mathrm{~mm}$ thick steel. The steel tape and the box are spot-welded together providing 
appropriate mechanical rigidity. The total length of the module corresponds to 5.7 nuclear interaction lengths. The individual module is capable to register the beam particle, which rate at CERN SPS is reaching $2 \times 10^{5}$ ions/sec with beam energy up to $150 \mathrm{AGeV}$, therefore no beam hole in the calorimeter is needed.
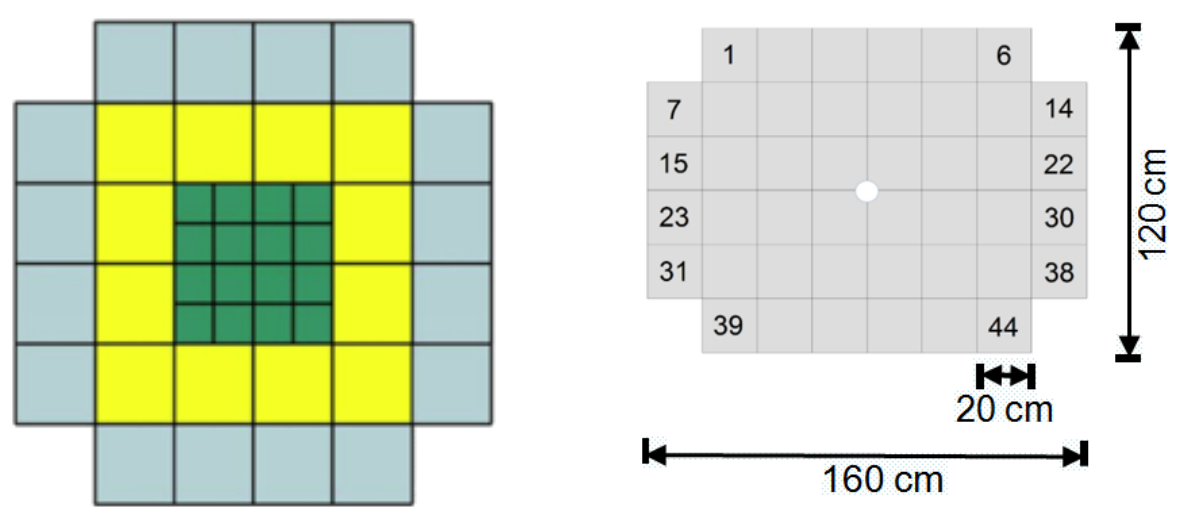

Figure 2: Transverse layout of the PSDs for the NA61/SHINE (left) and CBM (right).

The CBM PSD [3] has a similar design to that of the NA61/SHINE. The detector has 44 modules, each consisting of 60 lead/scintillator layers with a surface of $20 \times 20 \mathrm{~cm}^{2}$ (elongated transverse layout is illustrated in Fig. 2, right). The small $6 \mathrm{~cm}$ wide hole in the center of the CBM PSD is needed for the beam which at FAIR is expected to has a very high intensity up to $10^{9}$ ions/sec and energy up to $35 \mathrm{AGeV}$.

The Zero Degree Calorimeter (ZDC) for the MPD at NICA is also similarly designed [4]. The main differences are the number of modules (84), module size $\left(5 \times 5 \times 120 \mathrm{~cm}^{3}\right)$, and larger beam hole $\left(10 \times 10 \mathrm{~cm}^{2}\right)$. The transverse segmentation of ZDC fits the experimental conditions with beam intensities up to $10^{9}$ ions/sec and energy up to $6.3 \mathrm{AGeV}$.

Performance of the forward calorimeters is illustrated below based on the PSD module components R\&D and Monte-Carlo simulation of the CBM PSD detector response with realistic heavyion collision generators.

\section{Module response and radiation hardness of the detector components}

The energy resolution of the modules, linearity of the response, longitudinal shower profile, and compensation parameters were studied at CERN SPS/PS with muon, proton, and pion beams (contaminated by positrons). Figure 3(left) shows the assembled PSD module which was used for the tests. Figure 4 shows the energy resolution (left) and the linearity of the module response (right) for protons with momenta from 3 to $6 \mathrm{GeV}$. With the current design of the modules and the light readout scheme, the PSD response shows good linearity and the energy resolution of $\sigma_{E} / E<60 \% / \sqrt{E(\mathrm{GeV})}$.

Due to expected high intensities of the ion beams at FAIR it is important to study the radiation hardness of the calorimeter components. The total radiation dose of the PSD simulated with FLUKA [5] for the CBM geometry reaches $5 \times 10^{12}$ neutrons $/ \mathrm{cm}^{2}$ for two months of CBM 

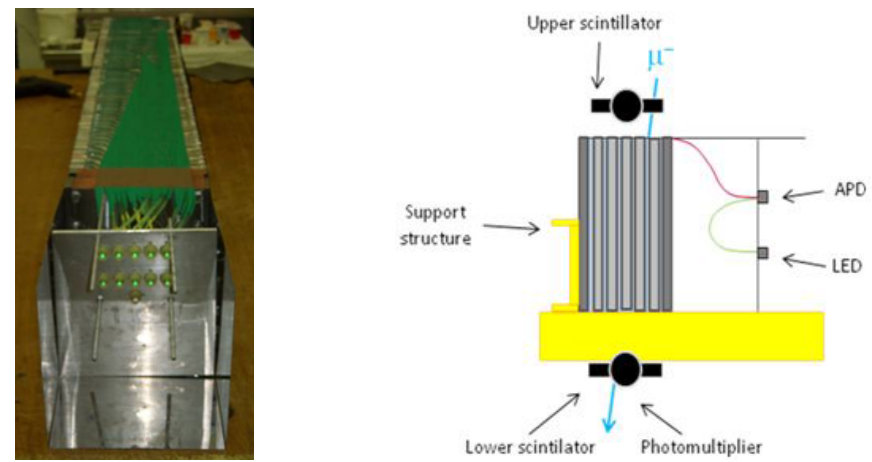

Figure 3: (left) An assembled PSD module. (right) Cosmic-rays setup for the APD tests.
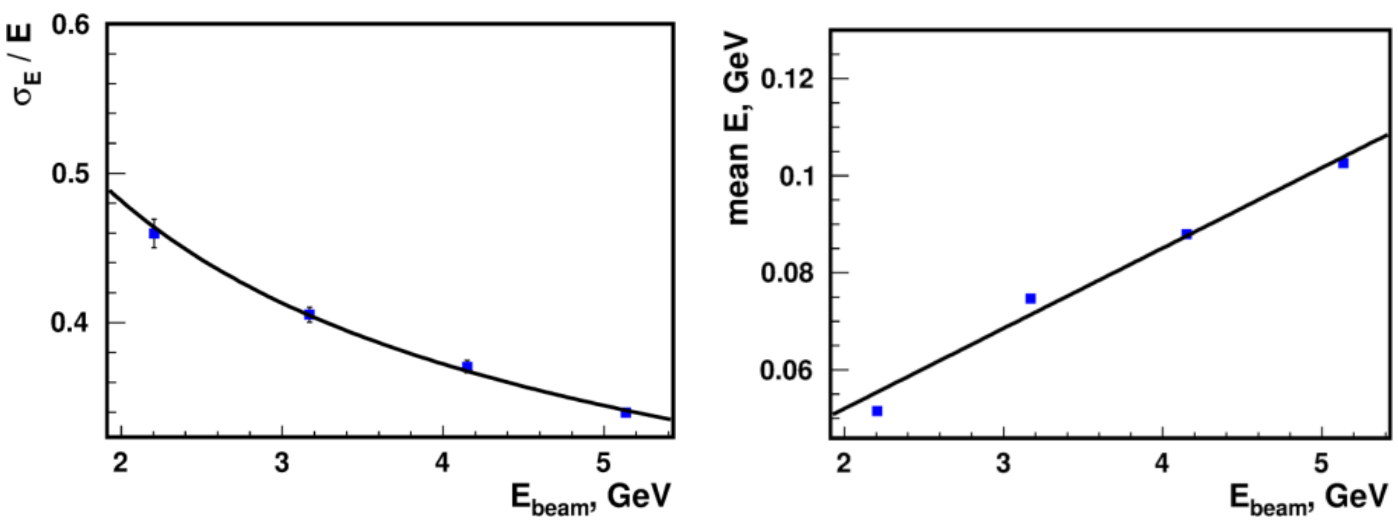

Figure 4: (left) Energy resolution and (right) linearity response to protons.

operation [3]. Such radiation dose is not critical for scintillators, but may significantly affect the avalanche photodiode (APD) performance.

The radiation hardness of the APDs were studied [6] with neutron fluxes up to $10^{13} \mathrm{n} / \mathrm{cm}^{2}$. The APDs produced by Hamamatsu (S12572-010P) and Ketek (PM3350) were irradiated at the Cyclotron facility of NPI Řež with a $35 \mathrm{MeV}$ secondary neutron beam with a dose about $10^{12} \mathrm{n} / \mathrm{cm}^{2}$ [7]. When irradiated with a 50 times smaller dose, the APD tests with cosmic muons (see Fig. 3(right) for the cosmic-rays setup) showed that signal and noise peaks are overlapping for the APD produced by Ketek, and are well separated for the APD produced by Hamamatsu. Further investigation revealed the dark current increase by a factor of $10^{3}$ after irradiation and that defects in silicon material can lead to increase of high frequency noise [8].

\section{Performance for centrality and reaction plane reconstruction}

The PSD performance for the collision centrality and the reaction plane reconstruction was studied with GEANT4 Monte-Carlo simulation of the CBM detector response and the DCMQGSM event generator [9] of heavy-ion collisions. The simulated CBM subsystems are shown in Fig. 5(left). They are a $250 \mathrm{~mm}$ thick Au target, eight silicon tracking stations (STS) located at 

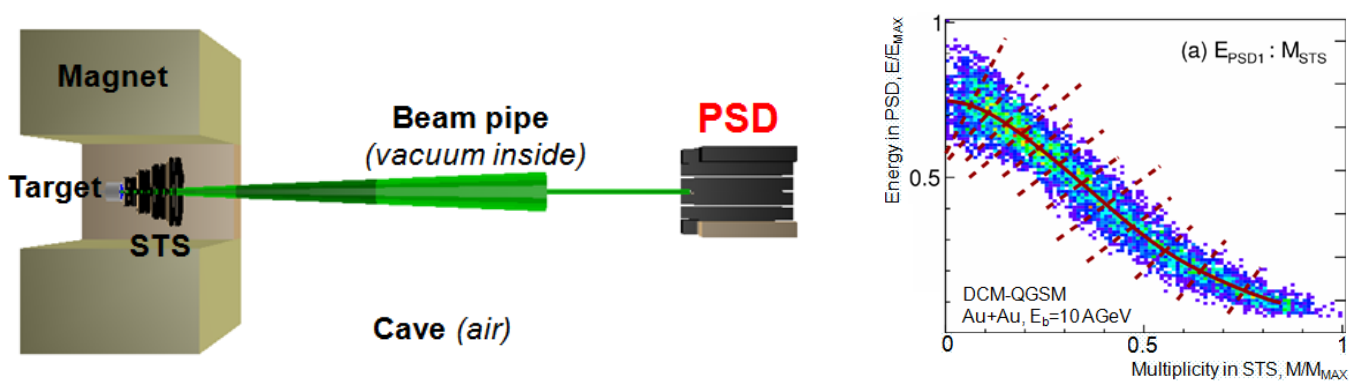

Figure 5: (left) The simulated CBM geometry. (right) Centrality determination procedure for CBM.

30-100 $\mathrm{cm}$ far from the target inside the dipole magnet, the aluminum beam pipe with a variable cross section and thickness of a few $\mathrm{mm}$, and the PSD. The distance of the PSD to the target was $8 \mathrm{~m}$ for beam energies $E_{b}=2-8 \mathrm{AGeV}$ and $15 \mathrm{~m}$ for $E_{b}=30 \mathrm{AGeV}$ [3].

The multiplicity of produced particles in the nuclei overlap zone was used as an experimental proxy of the impact parameter $b$ (or the number of participating nucleons) for a given heavy-ion collision. Figure 5(right) illustrates the centrality determination procedure for $\mathrm{Au}+\mathrm{Au}$ collisions at the beam energy $E_{b}=10 \mathrm{AGeV}$ [3]. Based on the correlation between the average energy in the PSD and the STS detector multiplicity the events were sorted in centrality classes of 5\%. The boundaries between centrality classes are shown by the dashed red lines in Fig. 5(right). The most right line corresponds to the most central event class. When used in a combination with the STS detector, the PSD helps to improve the overall centrality resolution in $0-40 \%$ range and allows for centrality determination in narrow centrality classes with a width of at least $5 \%$.

The azimuthal segmentation of the PSD in the plane transverse to the beam direction allows to measure the transverse kick to the spectators in the direction of the collision plane, and thus estimate the reaction plane angle. Figure 6(left) shows comparison between the reaction plane resolution
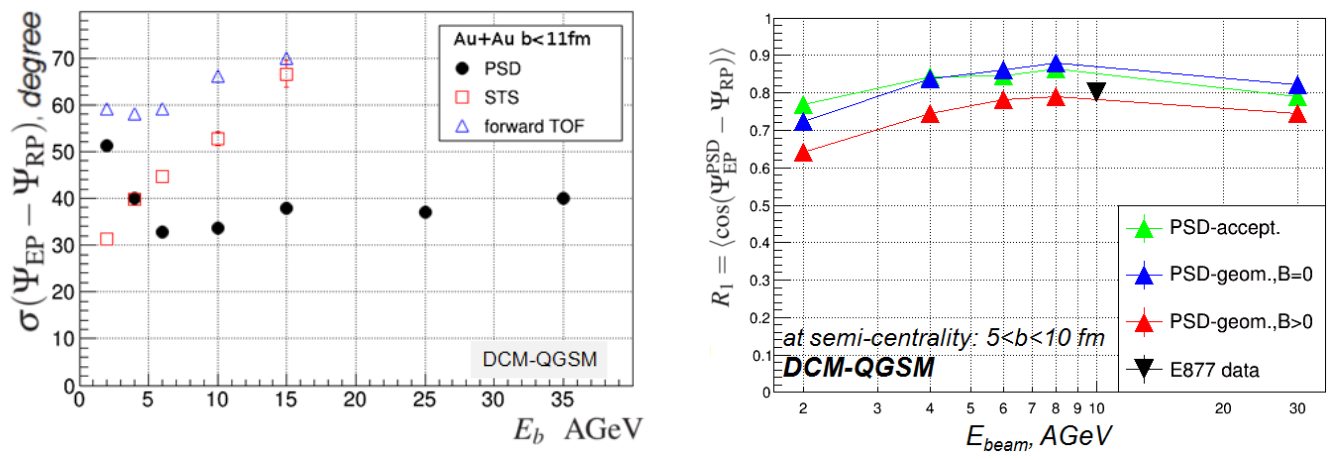

Figure 6: Reaction plane resolution correction factor vs. beam energy $E_{b}$. (left) Comparison between STS, TOF and PSD subsystems. (right) Results for different geometry configurations (particles in the PSD acceptance, simulations with and without magnetic field).

of the PSD, STS, and the forward time-of-flight (TOF) detector (using only information about azimuthal angles of the charged fragments in the PSD acceptance). The event plane resolution of 
the forward TOF is quite poor, and the STS shows good resolutions only up to $E_{b} \approx 4 \mathrm{AGeV}$. Above $E_{b}=4 \mathrm{AGeV}$ the PSD has a significantly better resolution. The results [10] of the simulations with other event generators, such as iQMD, UrQMD, LA-QGSM and HSD, yield in reaction plane resolution which is similar to the one obtained with the DCM-QGSM generator.

The PSD performance was also studied for the following three cases: (a) particle hits in the PSD acceptance $\left(0.215^{\circ}<\theta<5.0^{\circ}\right.$ for $E_{b}=2-8 \mathrm{AGeV}$ and $0.115^{\circ}<\theta<2.7^{\circ}$ for $\left.E_{b}=30 \mathrm{AGeV}\right)$, (b) simulations with magnetic field and (c) simulations without magnetic field. The resulting reaction plane resolution correction factor $R_{1}=\left\langle\cos \left(\Psi_{\mathrm{EP}}^{\mathrm{PSD}}-\Psi_{\mathrm{RP}}\right)\right\rangle$ simulated with the DCM-QGSM model is compared with E877 data in Fig. 6(right). The magnetic field results in a smaller value of the correction factor (worse reaction plane resolution) compared to the results with particle hits in the PSD acceptance which tests the effect of the transverse PSD granularity. In all cases the correction $R_{1}$ is in a good range of $0.6-0.8$ which is compatible to that of the E877 experiment [11].

\section{Summary}

Application of the compensating forward calorimeters for determination of the centrality and estimation of the reaction plane in a heavy-ion collisions at the CERN SPS, FAIR, and NICA experiments is discussed. Their good performance for such application is demonstrated based on simulations of the Projectile spectators Detector (PSD) for the future CBM experiment at FAIR. The performance study shows that combined with the information on particle multiplicity measured by the silicon tracking system, the PSD helps to improve the overall centrality determination in the range of $0-40 \%$, and reaction plane determination for beam energies above $4 \mathrm{AGeV}$.

The beam tests show good energy resolution and linearity of the PSD modules. The radiation hardness tests reveal that the most sensitive part of the PSD components are avalanche photodiodes. Their dark current increases after irradiation by a factor of $10^{3}$, and defects in silicon material lead to the increase of high frequency noise.

\section{Acknowledgments}

The authors thank the NPI cyclotron and neutron generators staff for excellent beam conditions and service. This work was supported by the European Community FP7 Capacities, contract HadronPhysics3 no.283286, LG12007 of the Ministry of Education of the Czech Republic and M100481202 of the Academy of Sciences of the Czech Republic grants, by project MSMT LG14004 of Cooperation Program between JINR and NPI in 2014-2015 and RFBR under grant No13-02-00101a, partially by TPU P.L.U.S. program.

\section{References}

[1] C. W. Fabjan and F. Gianotti, Rev. Mod. Phys. 75, 1243 (2003).

[2] N. Abgrall et al. [NA61 Collaboration], JINST 9, P06005 (2014) [arXiv:1401.4699 [physics.ins-det]].

[3] F. Guber et al. [CBM Collaboration], Technical Design Report for the CBM Projectile Spectator Detector (PSD), https://repository.gsi.de/record/109059. 
[4] V. D. Kekelidze et al. [NICA Collaboration], Design and Construction of Nuclotron-based Ion Collider fAcility. Conceptual Design Report, http://nica.jinr.ru.

[5] T.T. Böhlen et al., The FLUKA Code: Developments and Challenges for High Energy and Medical Applications, Nuclear Data Sheets 120, 211-214 (2014).

[6] V. Kushpil, V. Mikhaylov, V. P. Ladygin, A. Kugler, S. Kushpil, O. Svoboda and P. Tlustý, arXiv:1505.01297 [physics.ins-det].

[7] M. Štefánik et al., Neutron Spectrum Determination of the p(35 MeV)-Be Source Reaction by the Dosimetry Foils Method, Nuclear Data Sheets 119, 422 (2014).

[8] V. Mikhaylov et al., Radiation hardness tests of Avalanche Photodiodes for FAIR, NICA, and CERN SPS experiments, Proceedings of the EPS-HEP 2015 conference (2015).

[9] A. V. Dementyev and N. M. Sobolevsky, SHIELD, a Monte Carlo Hadron Transport Code, http://www.inr.troitsk.ru/shield/introd-eng.html.

[10] V. Mikhaylov et al., Particle flow and reaction plane reconstruction in the CBM experiment, Proceedings of the EPS-HEP 2015 conference (2015).

[11] J. Barrette et al. [E877 Collaboration], Phys. Rev. C 55, 1420 (1997) [Phys. Rev. C 56, 2336 (1997)] [nucl-ex/9610006]. 